\title{
STEFANIE NEUMEISTER
}

(Johanna-Stahl-Zentrum für jüdische Geschichte und Kultur in Unterfranken, Würzburg)

\author{
TRANSIT US-ZONE. ÜBERLEBENDE \\ DES HOLOCAUST IM BAYERN DER NACHKRIEGSZEIT \\ HG. V. S. STEINBACHER, \\ (DACHAUER SYMPOSIEN ZUR ZEITGESCHICHTE 13, 2013). \\ 248 S. ISBN: 978-3-8353-1344-6
}

Seit dem Jahr 2000 veranstaltet die Stadt Dachau in Zusammenarbeit mit dem Max Mannheimer Studienzentrum/Internationalen Jugendgästehaus jährlich eine Tagung, auf der aktuelle Forschungsansätze zur Geschichte der NS-Zeit diskutiert werden. 2012 beschäftigten sich die Teilnehmer des 12. Dachauer Symposiums zur Zeitgeschichte mit den jüdischen „Displaced Persons“ (DPs) in Bayern. Die zahlreichen Beiträge der Tagung sind nun in einem Sammelband erschienen.

1945 befanden sich auf dem Gebiet der drei westlichen Besatzungszonen 6,5 bis 7 Millionen DPs - Zivilpersonen, die sich kriegsbedingt außerhalb ihrer Heimat aufhielten. Eine vergleichsweise kleine Gruppe unter den DPs bildeten die jüdischen Überlebenden. Auf der Dachauer Tagung standen die Erfahrungen, die Lebensbedingungen und der Alltag der jüdischen DPs im Zentrum. Wie Sybille Steinbacher in ihrer Einführung hervorhebt, lebten im Herbst 1947 90\% aller jüdischen DPs in der US-amerikanischen Zone, meist waren sie in Lagern in Bayern untergebracht. Steinbacher verweist darauf, dass für die Mehrheit der 
jüdischen Überlebenden Deutschland nur ein „Transitzwischenraum“ (S. 8) gewesen sei, ein dauerhaftes Leben hier war für die meisten von ihnen jenseits allen Vorstellbaren.

Atina Großmann befasst sich in ihrem Beitrag mit dem „historischen Dreieck" der Beziehungen zwischen den jüdischen DPs, den amerikanischen Besatzern und den einheimischen Deutschen. Sie erläutert, wer die jüdischen DPs waren, von denen sich 1946/1947 rund 250000 in Deutschland aufhielten. Zum einen waren es Juden, die im Versteck gelebt oder sich als „Arier“ ausgegeben hatten, zum anderen waren es Überlebende von Arbeits-, Konzentrations- und Vernichtungslagern. Hinzu kamen Juden, die als Partner in einer sogenannten "privilegierten Mischehe" oder als Mischlinge überlebt hatten. Die bei Weitem größte Gruppe aber waren die osteuropäischen Juden, die aus deutschen Konzentrationslager kamen und solche, die zunächst nach Osteuropa zurückgekehrt waren, bis sie dort von einer erneuten heftigen Antisemitismuswelle überrascht wurden. Daraufhin kehrten viele von ihnen zurück nach Deutschland, um Schutz bei den amerikanischen Besatzern zu suchen. Obwohl für die meisten DPs feststand, dass Deutschland nur eine Zwischenstation war, bildete sich eine besondere Gemeinschaft heraus, die eigenen Komitees gründete und sich durch eine große kulturelle Vielfalt mit Synagogen, Schulen, Vereinen und Presseerzeugnissen auszeichnete. Großmann zeichnet weiterhin chronologisch die Entwicklung der politischen Rahmenbedingungen nach, die das Leben der DPs in Deutschland bestimmten. Interessant ist vor allem ihre Beobachtung, dass die Präsenz der DPs schon Ende 1947 nicht nur von den Deutschen als zunehmend lästiges Problem empfunden wurde, sondern auch von den Amerikanern. Während die USA auf eine Versöhnung mit Deutschland hinarbeitete, stand das Land selbst vor einer neuen großen Herausforderung: der Ankunft von 12 Millionen deutschen Flüchtlingen aus dem Osten. Die jüdischen DPs, die nicht weiterziehen konnten (aus finanziellen oder gesundheitlichen Gründen), waren nicht nur mit der Fremdenfeindlichkeit und den wieder aufflammenden alten Stereotypen der deutschen Bevölke- 
rung konfrontiert, sondern auch mit der äußerst heftigen Kritik von Seiten internationaler jüdischer Organisationen, die kein Verständnis für diejenigen hatten, die in Deutschland bleiben.

Auch Nikolaus Lehner gehörte zu denen, die blieben. Ihm ist der Tagungsband gewidmet, nicht zuletzt weil er sich über Jahrzehnte hinweg für die Erinnerungskultur und für die Jugendbegegnungsstätte Dachau eingesetzt hat. Barbara Distel umreißt in einem biographischen Artikel die wichtigsten Lebensstationen Lehners und seine Verdienste. Nikolaus Lehner, der aus Ungarn stammte, hatte Auschwitz und Dachau überlebt und war zunächst im DP-Lager Feldafing gestrandet. Nach vergeblichen Auswanderungsbemühungen ließ sich Lehner in Dachau nieder, wo er einen Holzverarbeitungsbetrieb eröffnete. In einem sehr persönlichen Beitrag schildert Juliana Alon ihre Erinnerungen an ihren Vater Nikolaus Lehner und an den schwierigen Umgang mit seinem Schweigen über die einschneidenden Erlebnisse, die sein Leben geprägt haben. Sie berichtet auch von den Schuldgefühlen des Vaters, die er mit zahlreichen Überlebenden teilte. Viele von ihnen konnten es nur schwer ertragen konnten, dass sie selbst gerettet wurden, während Millionen andere grausam sterben mussten.

Dirk Riedel stellt die größte Gruppe der Juden im Lagersystem Dachau vor, die Juden aus Ungarn. Sie unterschieden sich insofern von anderen Häftlingsgruppen, als dass sie keine gemeinsame Gruppenidentität besaßen, auch wenn sie im $\mathrm{KZ}$ als „ungarische Juden“ geführt wurden. Viele von ihnen stammten aus Gebieten, die erst während des Zweiten Weltkriegs im Rahmen von Annexionen zu Ungarn gekommen waren. Dazu gehörten die Juden aus der Süd- und Ostslowakei, aus der Karpatho-Ukraine, aus Nordsiebenbürgen, aus dem Übermurgebiet und der Batschka. 1944 begannen die Deportationen der Juden aus Ungarn, von denen die meisten nach Auschwitz-Birkenau gingen. Ein Teil der Juden wurde aber auch zur Zwangsarbeit eingesetzt, davon $5000 \mathrm{im}$ KZ Dachau und seinen Außenlagern. Riedel macht darauf aufmerksam, dass sich die Repatriierung der Juden aus Ungarn nach der Befreiung 1945 als besonders schwierig gestaltete. Durch den Zusammenbruch 
des ungarischen Regimes wurden die Annexionen rückgängig gemacht und für eine Vielzahl der sogenannten „ungarischen Juden“ stellte sich die Frage nach der Staatsbürgerschaft nun erneut. Daher zählten die Überlebenden aus Ungarn auch zu den letzten DPs, die nach Hause zurückgeschickt werden konnten.

Mit der Gemeinschaft der letzten Überlebenden, die sich selbst als „Scheerit Haplejta“ (dt. „der übriggebliebene Rest“) bezeichneten, beschäftigt sich Harold Marcuse. In seinem Text konzentriert er sich auf die jüdischen Überlebenden, die aus dem KZ Dachau und seinen Außenlagern befreit wurden. Diese hatten schon frühzeitig ein Selbstvertretungs-Komitee gegründet und kurz darauf war das Jewish Information Office entstanden, das sich um die Registrierung aller jüdischen Insassen bemühte, auch um die bereits verstorbenen. Ab Sommer 1945 verzeichnete das DP-Lager Dachau einen erneuten Zustrom von polnischen Juden, der eine Folge der fürchterlichen antisemitischen Massaker in Polen war und nach dem Pogrom von Kielce im Sommer 1946 noch weiter zunahm. Marcuse unterstreicht, dass auch für die zugewanderten polnischen Juden Deutschland lediglich eine Transitstation war auf dem Weg nach Palästina. Bereits im Juli 1945 trafen sich süddeutsche Delegierte aus verschiedenen DP-Lagern im Lager Feldafing und gründeten dort ein landesweites Komitee, das „Zentrale Komitee der Befreiten Juden in Bayern beziehungsweise der amerikanischen Zone" (ZK). Das ZK beschäftigte sich hauptsächlich mit Angelegenheiten der Auswanderung nach Palästina, war aber zugleich auch der wichtigste Ansprechpartner der US-Armee und der verschiedenen Hilfsorganisationen.

Jim G. Tobias, der sich schon seit mehreren Jahren der Erforschung der DP-Lager insbesondere in Franken widmet und eine kontinuierlich wachsende Internet-Datenbank zu den jüdischen DP-Lagern in der US-Zone ${ }^{1}$ etabliert hat, schildert die Lebensbedingungen der DPs in Dachau. Mit dem Umzug des Jewish Information Office vom DP-Lager

\footnotetext{
1 Vergleiche dazu die folgende Internetseite, unter: http://www.after-the-shoah.org.
} 
in die Stadt Dachau gründete sich auch die jüdische DP-Gemeinde Dachau. Neben seiner Hauptaufgabe, Familien wieder zusammenzuführen, war das Jewish Information Office vor allem darum bemüht, weitere Übernachtungsmöglichkeiten im Distrikt Dachau zu finden, da die Lager 1946 vollkommen überfüllt waren. Tobias betont, dass die DP-Gemeinde in Dachau eine sehr junge Gemeinschaft war, die schon bald die Hachschara-Idee wieder aufleben ließ. Bereits in den 1930er Jahren gab es in Deutschland mehrere Kibbuzim, in denen jüdische Jugendliche die Grundlagen des Ackerbaus und der Viehzucht erlernen konnten, um sich so auf ihre Emigration nach Palästina vorzubereiten. Diese Bewegung wurde 1945 erneut aufgegriffen. Meist waren es beschlagnahmte landwirtschaftliche Güter, die den Kibbuzniks zur Bewirtschaftung überlassen wurden. Der wohl bekannteste Hachschara-Kibbuz befand sich zwischen 1945 und 1948/1949 auf dem Pleikershof, dessen ehemaliger Besitzer Julius Streicher war, Herausgeber der antisemitischen Hetzschrift „Der Stürmer“.

Die Thematik der Kinderlager, auf die Tobias nur am Rande eingeht, wird von Andrea Andlauer in einem weiteren Beitrag vertieft. Das erste dieser Kinderzentren für jüdische und nichtjüdische Waisenkinder wurde im Juli $1945 \mathrm{im}$ Kloster Indersdorf eingerichtet. Von den 100-300 Kindern und Jugendlichen im Alter von 12-20 Jahren waren ein Drittel polnische und ungarische jüdische Kinder, ein Drittel waren verschleppte nichtjüdische Kinder und ein Drittel waren Kleinkinder aus Einrichtungen des Lebensborn e.V. Andlauer schildert sehr detailreich den Alltag in diesem Zentrum und stützt sich dabei vor allem auf die Aussagen von Greta Fischer, einer der leitenden Sozialarbeiterinnen der UNRRA (United Nations Relief and Rehabilitation Administration), die in dieser Einrichtung tätig war. Greta Fischer zeigte sich vor allem beeindruckt von dem Überlebenswillen der Kinder, den sie auch als eine „indiscribable rage to live“ (dt. „eine unbeschreibliche Wut zu leben“, S. 129) beschreibt. Dieser habe den Kindern geholfen, das Grauen der Lager zu überstehen. Diese unbändige Kraft, dieses daraus erwachsene Selbstbewusstsein, alle Hindernisse überwinden zu kön- 
nen, schien sich auch im Erwachsenenalter fortzusetzen. So jedenfalls konnte es Greta Fischer, die den Kontakt zu einigen der Waisenkinder über Jahre aufrechterhielt, immer wieder beobachten.

Ein Interviewprojekt aus den 1990er Jahren stellt Annette Eberle in ihrem Text vor, bei dem mehrere ehemalige DPs aus dem Lager Dachau zu ihren Erinnerungen an die Zeit unmittelbar nach ihrer Befreiung befragt wurden. Die Interviewten stammten alle aus Osteuropa und bemerkenswert ist, dass keiner von ihnen wieder in seine Heimat zurückgekehrt ist. Wie die Interviews laut Eberle gezeigt hätten, seien die meisten der Befragten nach der Befreiung zunächst gar nicht in der Lage gewesen, menschliche Gefühle wie Erleichterung oder Freude zu empfinden. Was sie aber antrieb, war der „der ausgeprägte Wunsch“ Wunsch, ihre Angehörigen wiederzufinden. Das zentrale Bezugssystem für die Überlebenden war stets die Familie, sowohl die verlorene, als auch später die neu gegründete. Außerdem sei in vielen Gesprächen deutlich geworden, dass von allen Überlebenden die Emigration angestrebt wurde, um möglichst weit weg von Deutschland zu sein. Obwohl sie sich sicher fühlten in der US-Zone - immerhin wurden sie hier versorgt und waren auch rechtlich anerkannt als Verfolgte -, wollte keiner von ihnen dauerhaft bleiben.

Mit dem Aufbau der jüdischen Nachkriegsgemeinschaft setzt sich Andrea Sinn auseinander. Ihr Blick ist vor allem auf die Institutionengeschichte gerichtet und auf die Rahmenbedingungen sowie die Probleme bei der Konsolidierung der jüdischen Gemeinschaft Anfang der 1950er Jahre. Zunächst hatten sich Landesverbände und DP-Komitees etabliert, doch es zeigte sich bald, dass die Interessen von DPs und deutschen Juden weit auseinander lagen, gerade im Hinblick auf die Frage „gehen oder bleiben“. Mit der Gründung des Zentralrats der Juden in Deutschland 1950 traten diese Gegensätze noch noch deutlicher zutage, zu groß waren die sprachlichen, religiösen und kulturellen Unterschiede zwischen deutschen und osteuropäischen Juden und zu verschieden die Zielsetzungen. Während sich der Zentralrat um den Wiederaufbau jüdischen Lebens in Deutschland bemühte, wollte die 
Mehrheit der osteuropäischen Juden nur eines, Deutschland schnellstmöglich den Rücken kehren.

Jürgen Zarusky beschäftigt sich in seinem Beitrag mit den viel diskutierten DP-Renten. Er legt dar, dass der bayerische Arbeitsminister im Jahr 1946 eine Verordnung erlassen hatte, wonach DPs, die in den DP-Lagern und zum Teil auch außerhalb der Lager arbeiteten, ebenso in die Sozialversicherung einzahlen sollten wie die übrige deutsche Bevölkerung. Heute streiten sich Sozialversicherer und Sozialgerichte um die Frage, ob jüdische DPs Anspruch auf eine Rente vom deutschen Staat haben. Aus seinen eigenen Erfahrungen als Gutachter in einem Gerichtsprozess, in dem es um die Rentenansprüche eines jüdischen Überlebenden aus Polen ging, berichtet Zarusky, dass die deutsche Rechtsprechung oft sehr oberflächlich mit historischen Quellen umgehe. Er kritisiert, dass die oft fehlende historische Kontextualisierung von Aussagen und Quellen zu falschen Schlüssen führe und damit auch zu Fehlurteilen.

Ein Interview mit dem Zeitzeugen Ernst Grube rundet den Tagungsband ab. Grube, dessen Mutter Jüdin und dessen Vater Kommunist war, gehörte zu den sogenannten „Geltungsjuden“. Er verbrachte eine Zeit lang in einem jüdischen Kinderheim und wurde mit 12 Jahren nach Theresienstadt deportiert. Grube überlebte die NS-Zeit und gelangte nach seiner Befreiung zunächst ins DP-Lager Dachau. Im Gespräch mit Jutta Neupert berichtet er von den Jahren unmittelbar nach seiner Befreiung und davon, wie wichtig ihm das Gefühl der $\mathrm{Zu}$ sammengehörigkeit war, insbesondere während der Schulzeit, ein Gefühl, das er vorher kaum gekannt habe. Auch auf die Gedenkkultur in Deutschland kommt Grube zu sprechen, die laut seiner Wahrnehmung erst um 1968-1970 allmählich in Bewegung kam. Vorher sei in der deutschen Öffentlichkeit kaum über die Opfer gesprochen worden. In Dachau hatte Grube, der sein Leben lang vehement für eine wachsende Erinnerungskultur eintrat, selbst immer wieder mit großen Widerständen zu kämpfen, so auch beim Aufbau der Jugendbegegnungsstätte. 
In der Zusammenschau bietet der vorliegende Tagungsband einen abwechslungsreichen und informativen Einblick in die Geschichte der DP-Lager in Bayern. Anzumerken ist jedoch, dass sich die meisten Beiträge auf das DP-Lager Dachau beziehen, weshalb der Titel ein wenig irreführend irreführend ist. In den nächsten Jahren werden hoffentlich zusätzliche Studien zu den zahlreichen weiteren Lagern in ganz Bayern folgen. Wie Norbert Frei in seinem Schlusswort schon festgestellt hat, ist auch die Rolle der Deutschen im Rahmen der Tagung nur schwach beleuchtet worden. Interessant wären daher weitere Untersuchungen zu den, wie er es nennt, Kontaktzonen (S. 234) zwischen den DPs und den Deutschen, gerade auch im Hinblick auf die persönlichen Beziehungen. 